\title{
La esencia de la comunicación en educación médica
}

\author{
X. Clèries
}

\begin{abstract}
La comunicación, entendida como relación humana, desempeña un papel esencial en los procesos de enseñanza, aprendizaje y evaluación de la dimensión comunicativa de las competencias profesionales en el ámbito de la salud. Las principales instituciones de educación médica han alcanzado consenso, avalado por evidencias, respecto a los objetivos, contenidos y metodologías tanto docentes como evaluativas que deben llevarse a cabo para un adecuado aprendizaje de habilidades de comunicación. Sin embargo, debido a la enorme complejidad de la comunicación humana en contextos de salud, enfermedad y trastornos, es necesario repensar los fundamentos epistemológicos adecuados para estudiar y evaluar la comunicación. El estudio y la evaluación deberían contemplarse en las realidades donde se interrelacionan profesionales y pacientes.
\end{abstract}

Palabras clave. Aprendizaje. Comunicación. Educación médica.

\section{The essence of communication in medical education}

The communication, understood as human relationship, plays an essential role in the processes of teaching, learning and assessing the communicative dimension of the professional competencies in the healthcare system. The main institutions of medical education have reached consensus, guaranteed by evidences, about the aims, contents and so much educational as evaluative methodologies that should be carry out for an adequate communication skills learning. Nevertheless, according the great complexity of the human communication in contexts of health, illness, disease and sickness, is necessary to rethink the best epistemological bases to study and to assess communication. The study and the evaluation should be contemplated in the real scenarios where there is professional-patient relationship.

Key words. Communication. Learning. Medical education.

\section{Introducción}

La palabra 'comunicación' se utiliza en la actualidad en múltiples contextos y con diversas polisemias que no siempre favorecen el entendimiento. Así, por ejemplo, a los medios de transporte (aéreos, terrestres, fluviales y marítimos) se les atribuye el sustantivo comunicación, como también a los medios de transmisión de información (televisión, radio, prensa e Internet). El modelo que ha acabado imperando al referirse a la comunicación es el que proviene del ámbito de la mecánica y de la ingeniería, y que tuvo su auge con el desarrollo del telégrafo, basado en un emisor que emite un mensaje a un receptor a través de un canal y mediante un código [1]. Sin embargo, existen otros modelos que explican el fenómeno de la comunicación desde una perspectiva que tiene en cuenta la relación entre seres humanos y que concibe la transmisión de mensajes de forma helicoidal [2,3] frente a la representación lineal del modelo mecanicista citado con anterioridad. Probablemente este tipo de modelos sean más adecuados para contemplar en el ámbito de la educación médica, donde además de transmitir mensajes lo suficientemente inteligibles, también es preciso tener en cuenta la naturaleza humana en procesos de enseñanza $\mathrm{y}$ aprendizaje.

El presente artículo pretende señalar el papel esencial que la comunicación desempeña en la competencia de las profesiones sanitarias, en los procesos de enseñanza y aprendizaje de estos profesionales, y en la evaluación de la dimensión comunicativa de la competencia de los profesionales de la salud.

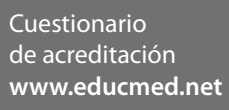

www.educmed.net

Institut d'Estudis de la

Salut. Generalitat de

Catalunya. Barcelona,

España.

Correspondencia

Dr. Xavier Clèries Costa

Institut d'Estudis de la

Salut. Roc Boronat, 81-95.

E-08005 Barcelona.

E-mail

fx.cleries@gencat.cat

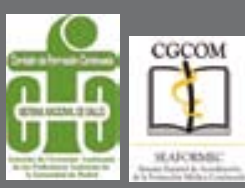

Actividad acreditada por SEAFORMEC con 1,5 créditos

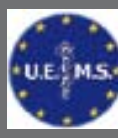

Créditos reconocidos por el'European

Accreditation Council for CME' de la UEMS 


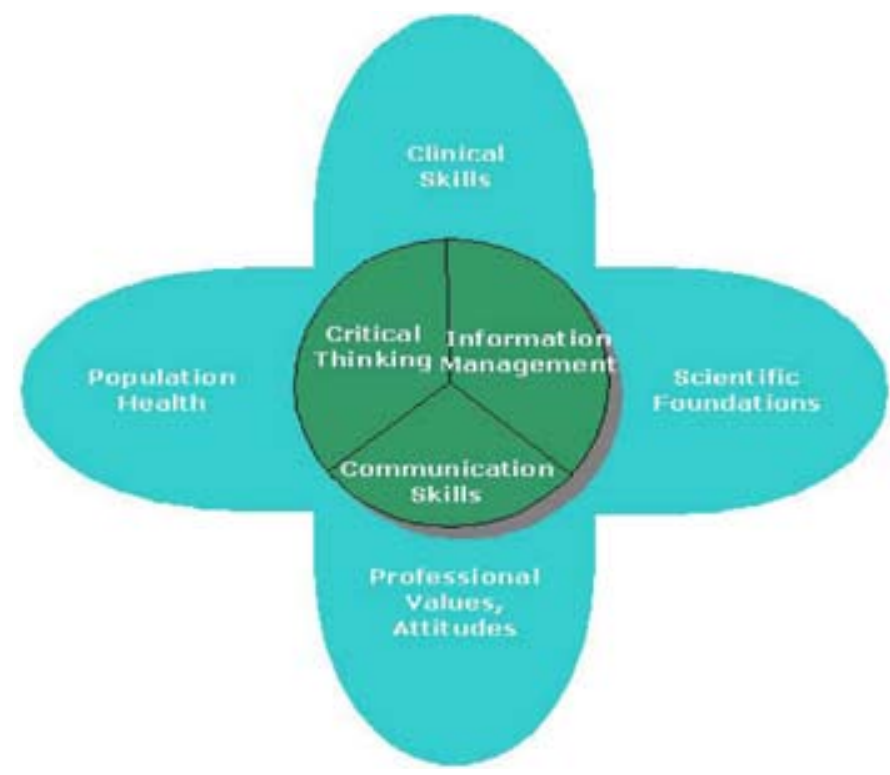

Figura 1. Dimensiones esenciales de la competencia. Global Minimum Essential Requirements (Institute for International Medical Education). Disponible en: http://www.iime.org/gmer.htm.

\section{Competencia de los profesionales sanitarios y comunicación}

Desde principios de la década de los noventa se define la competencia profesional como la capacidad de utilizar los conocimientos y aptitudes para resolver los problemas que se presentan en el ejercicio de la práctica cotidiana y poder afrontar diferentes situaciones propias de su disciplina [4]. En la actualidad no existe duda alguna -al menos desde las principales instituciones, nacionales [5-7] e internacionales [8-11], dedicadas a la educación médica- que la comunicación constituye una de las partes nucleares de la competencia de la profesión médica (y también extensible al resto de las profesiones sanitarias) (Fig. 1).

Así pues, se puede aseverar con rotundidad que la comunicación no es algo que depende de la manera de ser de cada profesional o una dimensión secundaria frente a las dimensiones clínicas. Actualmente se considera la comunicación como una dimensión de la competencia de las profesiones sanitarias susceptible de enseñarse, aprenderse y evaluarse. Estos aspectos, incluso, vienen avalados por la evidencia de estudios que demuestran que una adecuada comunicación repercute en una mayor confianza en la relación médico-paciente, en una mayor adhesión terapéutica por parte del paciente, en una disminución de las prescripciones terapéuticas, en mejores resultados clínicos en el curso de las enfermedades y en una disminución de las demandas contra los profesionales [12-15].

\section{Enseñanza, aprendizaje y evaluación de la comunicación}

La enseñanza de las habilidades de comunicación posee una relativamente corta existencia en la enseñanza de pregrado (actualmente de grado) de medicina y desigual fortuna en el resto de enseñanzas de ciencias de la salud. Por lo que respecta a medicina, los inicios se sitúan en la década de los setenta en el Reino Unido, Estados Unidos y Canadá. Sin embargo, es en el primer decenio del siglo XXI cuando se estructuran unas bases conceptuales y metodológicas sólidas respecto el aprendizaje de comunicación de los futuros profesionales de la salud $[16,17]$ y que de manera esquemática se reflejan en la figura 2. Cabe destacar que, además de las habilidades comunicativas y de entrevista clínica con el paciente, se tienen en cuenta también las actitudes (respeto a los demás), la capacidad de dominar otros medios de comunicación (escritos, virtuales), la gestión de la incertidumbre y de las emociones, 


\section{Programa de Formación Médica Acreditada en Educación Médica}

Artículo: La esencia de la comunicación en educación médica Autor: X. Clèries

Revista: Educ Med 2010; 13: 25-31

\section{Según el Institute for International Medical Education, ¿Cuál de las siguientes dimensiones no es esencial en la competencia profesional médica?:}

$\square$ a) Las habilidades clínicas

$\square$ b) Las habilidades comunicacionales

$\square$ c) Las habilidades de redacción escrita

$\square$ d) La salud pública

$\square$ e) El pensamiento crítico

\section{2. ¿Qué instrumento para evaluar habilidades de} comunicación ha demostrado ser mas eficiente?

$\square$ a) Depression Rating Scale de Hamilton

$\square$ b) The Segue Framework de Makoul et al

$\square$ c) La guía de habilidades sociales de Vicente Caballo

$\square$ d) La entrevista motivacional de Francesc Borrell

$\square$ e) La entrevista clínica de Freud

3. La competencia profesional, según Kane, se define como:

$\square$ a) La capacidad de utilizar los conocimientos y aptitudes para resolver los problemas que se presentan en el ejercicio de la práctica cotidiana y poder afrontar diferentes situaciones propias de su disciplina

$\square$ b) La capacidad de aprender y reflexionar sobre la realidad

$\square$ c) La capacidad de adquirir conocimientos, habilidades y actitudes sobre las actividades diarias características de la vida laboral

$\square$ d) La capacidad de identificar incidentes críticos en el ejercicio de la profesión

$\square$ e) La integración de conocimientos, actitudes, habilidades y aptitudes para afrontar los problemas de la vida laboral

\section{En el currículo de formación de grado de medicina} de las facultades de medicina del Reino Unido, según von Fragstein et al y respecto a la enseñanza de comunicación, se tienen en cuenta valores como:

$\square$ a) El compañerismo

$\square$ b) La simpatía

$\square$ c) El profesionalismo

$\square$ d) El humanismo

$\square$ e) La sinceridad

\section{Las ECOE constituyen una metodología en la que se} evalúan, entre otras dimensiones, la comunicación en estudiantes y profesionales de la salud. Pero, ¿qué significan las siglas ECOE?:

$\square$ a) Evaluación compleja objetiva estructurada

$\square$ b) Evaluación clínica objetiva especializada

$\square$ c) Evaluación clínica objetiva enseñada

$\square$ d) Evaluación conjunta objetiva estructurada

$\square$ e) Evaluación clínica objetiva estructurada

\section{Encontrará este cuestionario} en la web de la revista: www.educmed.net

Nombre y apellidos.

6. Según Edgard Morin, para que un conocimiento sea pertinente es necesario que sea:

$\square$ a) Contextualizado y comprenda la globalidad y la multidimensionalidad

$\square$ b) Construido en un contexto metadimensional

$\square$ c) Perfectamente enseñado y puesto en práctica

$\square$ d) Verificable y replicable

$\square$ e) Cooperativo y multidimensional

7. Los paradigmas interpretativos (o constructivistas):

$\square$ a) Pretenden describir y comprender el objeto de estudio

$\square$ b) Determinan la objetividad del objeto de estudio

$\square$ c) Clasifican y establecen prioridades del objeto de estudio

$\square$ d) Infieren sólo las características observables y medibles del objeto de estudio

$\square$ e) Todas son falsas

8. ¿Cuál de los siguientes instrumentos no es adecuado para el aprendizaje de comunicación?:

$\square$ a) El self-audit

$\square$ b) El peer-review

$\square$ c) La confrontación dialéctica

$\square$ d) El portafolio

$\square$ e) El role playing

9. La escala CICAA (Conectar, Identificar, Comprender, Acordar, Ayudar) es un instrumento que sirve para:

$\square$ a) Evaluar las actitudes cordiales de los profesionales de la salud

$\square$ b) Identificar los problemas de comunicación de los médicos con los pacientes difíciles

$\square$ c) Enseñar a comunicar a los profesionales de la salud

$\square$ d) Subsanar los fallos de relación de los médicos con los pacientes depresivos

$\square$ e) Evaluar la comunicación clínica en las consultas

10. La American Academy on Communication in Healthcare es una institución que tiene por finalidad:

$\square$ a) Velar por el cumplimiento de los derechos de los pacientes

$\square$ b) La integración de conocimientos de comunicación y su aplicación en el ámbito de la salud desde la vertiente formativa, investigadora y divulgativa

$\square$ c) Evaluar las competencias de los profesionales de la medicina

$\square$ d) Enseñar a los pacientes a relacionarse mejor con los médicos

$\square$ e) Mediar en los conflictos que surgen entre médicos y pacientes

DNI

E-mail.

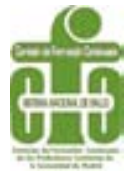




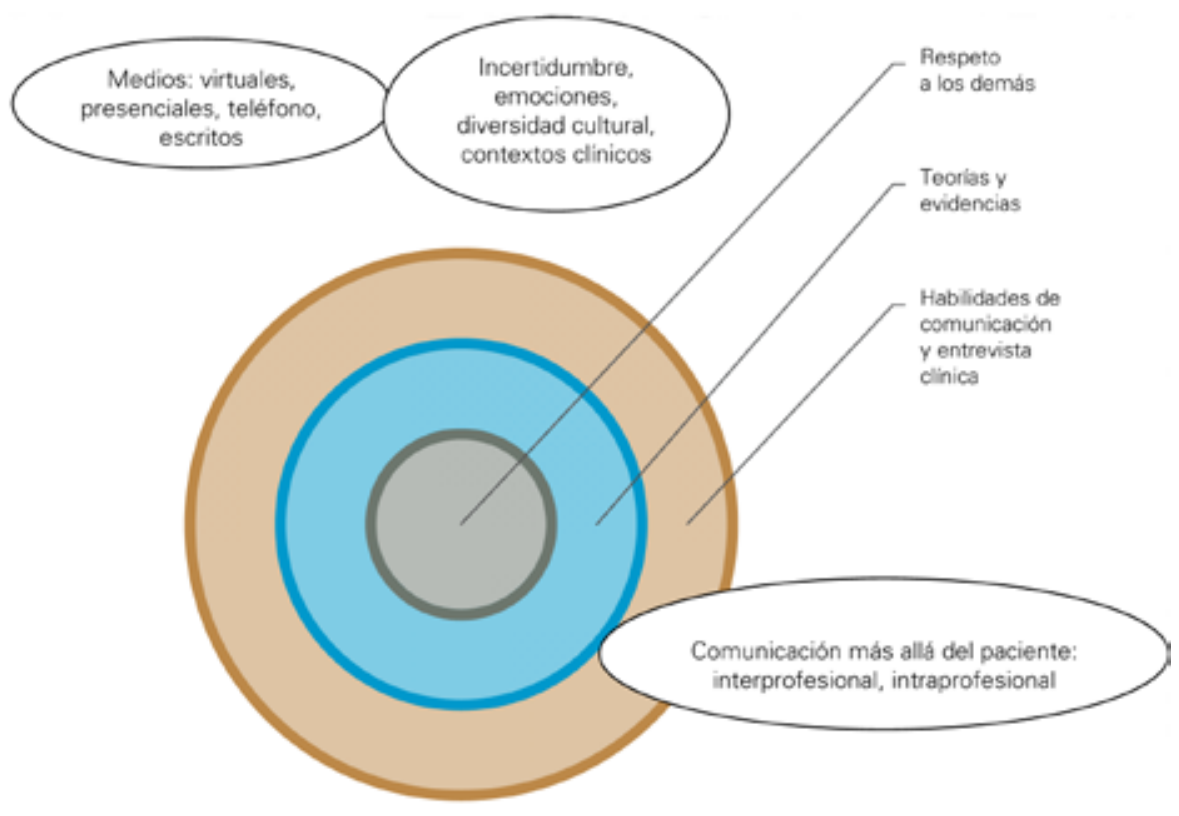

Profesionalismo

Principios éticos y legales

Reflexión en la práctica

Evidencia basada en la práctica

Figura 2. Currículo de comunicación clínica en el grado de educación médica (adaptado de [17]).

los valores (ética, profesionalismo, reflexión) y las relaciones interprofesionales.

La relación asistencial con el paciente, no obstante, constituye el marco en el que se ha desarrollado el mayor bagaje de objetivos, contenidos y metodología respecto al aprendizaje de comunicación con referentes nacionales $[18,19]$ e internacionales [20] de gran prestigio. La especificidad de qué y cómo enseñar comunicación se refleja en la tabla I. Es interesante anotar que, en nuestro contexto, la enseñanza de habilidades de comunicación y entrevista clínica ha estado ligada al desarrollo de la especialidad de medicina familiar y comunitaria, habiendo adoptado un eficaz pragmatismo [21]. Las técnicas e instrumentos que han demostrado ser más eficaces y eficientes en el aprendizaje de comunicación ( $y$, en general, en la educación médica) son el feedback docente, el role playing sin y con pacientes simulados y la observación y videograbación de la consulta con pacientes reales, a través de actividades formativas (cursos, seminarios, talleres) con un número reducido de alumnos (máximo de 15 personas) [18-22].
Por otro lado, la evaluación es un aspecto que debe observarse como integrado en el aprendizaje de comunicación en educación médica. Los métodos de evaluación de la dimensión comunicativa de la competencia de los profesionales vienen determinados por la observación directa y la utilización de checklists, ya sea en entrevistas con pacientes reales o mediante la simulación con pacientes estandarizados. Los principales instrumentos evaluativos validados de la comunicación pueden observarse en la tabla II.

Respecto a la utilización de pacientes estandarizados, una de las experiencias más celebradas en nuestro contexto es la que ha llevado a cabo el Institut d'Estudis de la Salut (IES), de la Generalitat de Catalunya, a través de la evaluación clínica objetiva estructurada (ECOE) de los profesionales sanitarios [23], con el asesoramiento de la Educational Commission for Foreign Medical Graduates (ECFMG) del National Board of Medical Examiners (NBME) estadounidense y del Centre d'Évaluation des Sciences de la Santé de l'Université de Laval, en Québec (Canadá). 


\section{Reflexiones y perspectivas de futuro}

Las principales evidencias en referencia al aprendizaje de comunicación se concentran mayoritariamente en las habilidades de comunicación en la enseñanza de grado de medicina del denominado 'mundo anglosajón'. En nuestro contexto continúa siendo testimonial. Sin embargo, la comunicación humana, tal y como se ha planteado anteriormente en la introducción, es de tal complejidad que más allá de lo que es observable, las habilidades de comunicación, también intervienen los aspectos contextuales, la globalidad y la multidimensionalidad (biopsicosocial), en la línea propuesta por Edgard Morin para que un conocimiento sea pertinente [24]. Aplicado al ámbito de la salud, se debería cuestionar si la comunicación es más que una habilidad, teniendo que contemplar, además, las actitudes y la capacidad de autorreflexión [25].

En este sentido, la enseñanza y la evaluación de comunicación en el ámbito de la salud tienen aún un amplio recorrido al tener que generar alternativas acordes con las características de la interrelación que se da en la comunicación asistencial. Así, por ejemplo, es cuestionable el acercamiento ontológico y epistemológico con el que se ha venido abordando la evaluación de la comunicación médico-paciente, basado en un paradigma positivista que pretende explicar, controlar y predecir, desde la atalaya privilegiada de los expertos y en aras a la objetividad, algo tan complejo como las relaciones interpersonales [26]. Todo ello en detrimento de paradigmas interpretativos (o constructivistas), que pretenden describir y comprender el objeto de estudio que, en el caso que nos ocupa, está determinado por personas que desarrollan diferentes roles: docentes, alumnos, pacientes, profesionales y pacientes. Quizás este tipo de paradigmas ofrecen una plataforma epistemológica más idónea para aproximarse a la complejidad de la comunicación humana. El paradigma interpretativo concibe una realidad múltiple, la peculiaridad de cada caso, la contextualización de los fenómenos, la subjetividad, el análisis de situaciones particulares y la realidad en su totalidad y complejidad. Probablemente, la metodología cualitativa constituya una opción alternativa, y por supuesto complementaria, a la cuantitativa, y no por ello exenta de rigurosidad científica. Es más, hallazgos obtenidos mediante la metodología cualitativa pueden llegar a facilitar posteriores estudios metodológicamente cuantitativos $[27,28]$ y, por qué no, la elaboración de esca-
Tabla I. Conceptos, contenidos y metodología en el aprendizaje de comunicación [18-20,22].

Conceptos de aprendizaje

- Definición clara de las habilidades esenciales, fundamentada en evidencia

- Demostración de las habilidades que se tienen que aprender

- Observación de entrevistas realizadas por el estudiante

- Feedback descriptivo y constructivo

- Práctica en un ambiente seguro

- Evaluación-autoevaluación/introspección

Contenidos básicos

- Estructura y contenido de la entrevista clínica

- Establecimiento de la relación con el paciente

- Inicio del encuentro

- Recogida de información

- Entender la perspectiva del paciente

- Compartir información

- Alcanzar un acuerdo con el paciente

- Cierre de la entrevista

Contenidos avanzados

- Gestión de emociones

- Aspectos culturales

- Promover conductas saludables y favorecer la adhesión terapéutica

- Toma de decisiones clínicas compartidas Exploración de aspectos sexuales y religiosos

Comunicación en situaciones específicas

- Entrevista familiar

- Entrevista pediátrica y con adolescentes

- Entrevista geriátrica

- Problemática en el ámbito de la salud mental

- Violencia de género

- Problemática relacionada con adicciones

- Enfermedades minoritarias

- Dar malas noticias

- Comunicar errores médicos

Comunicación con otros colegas

- Presentaciones orales

- Trabajo en equipo

- Docencia

Metodología docente

- Grupos reducidos

- Uso del role playing

- Simulaciones con pacientes estandarizados

- Filmación de role playing, simulaciones y entrevistas reales

- Reflexión 
Tabla II. Principales instrumentos de evaluación de la comunicación.

\begin{tabular}{|c|c|c|}
\hline Instrumento & Referencia & Descripción \\
\hline $\begin{array}{l}\text { Calgary-Cambridge } \\
\text { Observation Guide }\end{array}$ & $\begin{array}{l}\text { Kurtz S, et al. } \\
\text { Acad Med 2003; 78: 802-9 }\end{array}$ & $\begin{array}{l}71 \text { ítems sobre aspectos observables en la entrevista } \\
\text { clínica: iniciar la sesión, obtener información, construir } \\
\text { la relación, explicar y planificar, y cerrar la sesión }\end{array}$ \\
\hline $\begin{array}{l}\text { Kalamazoo Essential Elements: } \\
\text { The Communication Checklist }\end{array}$ & $\begin{array}{l}\text { Makoul G. } \\
\text { Acad Med 2001; 76: 390-3 }\end{array}$ & $\begin{array}{l}\text { Contempla } 22 \text { áreas de contenidos relacionados con la } \\
\text { entrevista clínica; utiliza una escala de Likert de } 4 \text { puntos }\end{array}$ \\
\hline $\begin{array}{l}\text { MASS-Global Rating List for } \\
\text { Consultation Skills of Doctors }\end{array}$ & $\begin{array}{l}\text { Van Thiel J, et al. } \\
\text { Med Educ 1991; 25: 224-9 }\end{array}$ & $\begin{array}{l}\text { Evalúa } 17 \text { habilidades mediante una escala de Likert } \\
\text { de } 8 \text { puntos }\end{array}$ \\
\hline $\begin{array}{l}\text { Macy Model Checklist-Case } \\
\text { Western Reserve University }\end{array}$ & $\begin{array}{l}\text { Kalet A, et al. } \\
\text { Acad Med 2004; 79: 511-20 }\end{array}$ & $\begin{array}{l}57 \text { áreas en relación a la entrevista clínica; escala de } \\
\text { Likert de } 4 \text { puntos }\end{array}$ \\
\hline The SEGUE Framework & $\begin{array}{l}\text { Makoul G. Patient Educ } \\
\text { Couns 2001; } 45: 23-34\end{array}$ & $\begin{array}{l}6 \text { áreas referentes a entrevista clínica con respuesta } \\
\text { dicotómica sí/no }\end{array}$ \\
\hline $\begin{array}{l}\text { Escala CICAA (Conectar, } \\
\text { Identificar, Comprender, } \\
\text { Acordar, Ayudar) }\end{array}$ & $\begin{array}{l}\text { Ruiz-Moral R y Pérula de } \\
\text { la Torre LA. Aten Primaria } \\
\text { 2006; 37: 320-4 }\end{array}$ & $\begin{array}{l}36 \text { ítems relacionados con la entrevista clínica: conectar, } \\
\text { identificar y comprender los problemas, acordary ayudar; } \\
\text { evaluación mediante escala de Likert de } 3 \text { puntos }\end{array}$ \\
\hline $\begin{array}{l}\text { Pacientes estandarizados } \\
\text { y reales }\end{array}$ & $\begin{array}{l}\text { Whelan GP. Med Teach } \\
\text { 1999; 21: 156-60 }\end{array}$ & $\begin{array}{l}4 \text { áreas de entrevista clínica mediante escala de Likert } \\
\text { de } 4 \text { puntos }\end{array}$ \\
\hline $\begin{array}{l}\text { Pacientes estandarizados } \\
\text { y reales }\end{array}$ & $\begin{array}{l}\text { Epstein RM, et al. Teach } \\
\text { Learn Med 2004; 16: 196-9 }\end{array}$ & $\begin{array}{l}16 \text { preguntas sobre entrevista clínica mediante escala } \\
\text { de Likert de } 6 \text { puntos }\end{array}$ \\
\hline
\end{tabular}

las de evaluación de la comunicación a partir de la realidad de los principales actores y no desde la perspectiva de los expertos.

La reflexión, el autoconocimiento y la autoevaluación devienen, entre otros, procesos personales que están demostrando un papel crucial en el aprendizaje y el desarrollo profesional [29-31]. Estos procesos metodológicos generan instrumentos como el self-audit, el peer-review y el portafolio que, con la debida rigurosidad, deben desempeñar una importante baza en el aprendizaje y la evaluación de la comunicación.

En definitiva, las perspectivas de futuro de la comunicación en educación médica invitan a desarrollar diferentes ámbitos de trabajo, tanto en la formación de grado, posgrado y continuada, como en la investigación. Los profesionales de la salud están llamados en primera instancia a adquirir adecuadamente la dimensión comunicativa de sus competencias. Pero no hay que olvidar a otros actores principales del ámbito sanitario, como son los pacientes, sus familiares y la ciudadanía, que también son susceptibles de recibir los beneficios de la comunicación en educación médica. La integración de conocimientos de diversas disciplinas y su aplicación en amplios segmentos de la población, en su condición de profesionales y pacientes, desde la vertiente formativa, investigadora y divulgativa, forma parte de la finalidad de instituciones como la European Association for Communication in Healthcare (http://www. each.nl) y la American Academy on Communication in Healthcare (http://www.aachonline.org), que están dando ejemplo del gran alcance de la comunicación en educación médica.

\section{Bibliografía}

1. Shannon C, Weaver E. The mathematical theory of communication. Chicago: University of Illinois Press; 1949.

2. Dance FEX. Hacia una teoría de la comunicación humana. In Dance FEX, ed. Teoría de la comunicación humana: ensayos originales. Buenos Aires: Troquel; 1973. p. 403. 
3. Watzlawick P, Beavin J, Jackson DD. Teoría de la comunicación humana. 8. ed. Barcelona: Herder; 1991.

4. Kane MT. The assessment of professional competence. Education and the Health Professions 1992; 15: 163-82.

5. SEDEM (Sociedad Española de Educación Médica); ACEM (Associació Catalana d'Educació Mèdica); SEMDE (Sociedad de Educación Médica de Euskadi); SADEM (Sociedad Aragonesa de Educación Médica). Recomendaciones para un nuevo proceso de reforma curricular en las facultades de medicina españolas. Educ Med 2005; 8: 3-7.

6. ANECA (Agencia Nacional de Evaluación de la Calidad y Acreditación). Libro blanco. Título de grado en Medicina, Odontología, Enfermería, Psicología y Farmacia. URL: http://www.aneca.es/media/150312/libroblanco_medicina_def.pdf. URL: http://www.aneca.es/media/150344/ libroblanco_odontologia_def.pdf. URL: http://www. aneca.es/media/150360/libroblanco_jun05_enfermeria. pdf. URL: http://www.aneca.es/media/150356/libroblanco_psicologia_def.pdf. URL: http://www.aneca.es/ media/150368/libroblanco_farmacia_def.pdf.

7. AQU Catalunya. Competències professionals bàsiques comunes dels llicenciats en Medicina formats a les universitats de Catalunya. Barcelona: Agència per a la Qualitat del Sistema Universitari de Catalunya; 2004. URL: http://www.medab.uclm.es/publico/master/m1/ Requeriments\%20globals\%20minims\%20educacio\%20 medicina-pdf.

8. General Medical Council. Review of 'Tomorrow's doctors'; 2008. URL: http://www.gmc-uk.org/news/articles/ review\%20of\%20tomorrow.pdf.

9. The Scottish Dean's Medical Education Group. The Scottish doctor. Learning outcomes for the medical undergraduate in Scotland: a foundation for competent and reflective practitioners. 3 ed. Dundee: Association for Medical Education in Europe (AMEE); 2008.

10. Swing SR. The ACGME outcome project: retrospective and prospective. Med Teach 2007; 29: 648-54.

11. Hamilton JD. International standards of medical education: a global responsibility. Med Teach 2008; 22: 547-8.

12. Stewart MA, Brown JB, Donner A, McWhinney IR, Oates J, Weston WW, et al. The impact of patient-centered care on outcomes. J Fam Pract 2000; 49: 796-804.

13. DiMatteo MR, Haskard KB, Williams SL. Health beliefs, disease severity, and patient adherence: a meta-analysis. Med Care 2007; 45: 521-8.

14. Tamblyn R, Abrahamowicz M, Dauphinee D, Wenghofer E, Jacques A, Klass D, et al. Physician scores on a national clinical skills examination as predictors of complaints to medical regulatory authorities. JAMA 2007; 298: 993-1001.
15. Street RL, Makoul G, Arora NK, Epstein RM. How does communication heal? Pathways linking clinician-patient communications to health outcomes. Patient Educ Couns 2009; 74: 295-301.

16. Makoul G. Essential elements of communication in medical encounters: the Kalamazoo consensus statement. Acad Med 2001; 76: 390-3.

17. Von Fragstein M, Silverman J, Cushing A, Quilligan S, Salisbury H, Wiskin C; UK Council for Clinical Communication Skills Teaching in Undergraduate Medical Education. UK consensus statement on the content of communication curricula in undergraduate medical education. Med Educ 2008; 42: 1100-7.

18. Borrell F. Entrevista clínica: manual de estrategias prácticas. Barcelona: SemFYC; 2004.

19. Ruiz R. Relación clínica. Guía para aprender, enseñar e investigar. Barcelona: SemFYC; 2004.

20. Kurtz S, Silverman J, Draper J. Teaching and learning communication skills in medicine. Abingdon: Radcliffe Medical Press; 1998.

21. Clèries X, Borrell F, Epstein RM, Kronfly E, Escoda JJ, Martínez-Carretero JM. Aspectos comunicacionales: el reto de la competencia de la profesión médica. Aten Primaria 2003; 32: 110-7.

22. Ruiz R. Educación Médica. Manual práctico para clínicos. Madrid: Médica Panamericana; 2010.

23. Kronfly E, Ricarte JI, Juncosa S, Martínez-Carretero JM. Evaluación de la competencia clínica de las facultades de medicina de Cataluña, 1994-2006: evolución de los formatos de examen hasta la evaluación clínica objetiva y estructurada (ECOE). Med Clin (Barc) 2007; 129: 777-84.

24. Morin E. Los siete saberes necesarios para la educación del futuro. París: UNESCO; 1999.

25. Zoppi K, Epstein RM. Is communications a skill? Communication behaviors, and being in relation. Fam Med 2002; 34: 319-24.

26. Sarrado JJ, Clèries X, Ferrer M, Kronfly E. Evidencia científica en medicina: ¿única alternativa? Gac Sanit 2004 18: 235-44.

27. Cook TD, Reichardt CS. Métodos cualitativos y cuantitativos en investigación evaluativa. Madrid: Morata; 1986.

28. Steekler A, McLeroy KR, Goodman RM. Towards integrating qualitative and quantitative methods: an introduction. Health Educ Q 1992; 19: 1-8.

29. Epstein RM. Assessment in medical education. N Engl J Med 2007; 356: 387-96.

30. Epstein RM. Reflection, perception and acquisition of wisdom. Med Educ 2008; 42: 1048-50.

31. Roma J. La reflexión como eje central del desarrollo profesional. Educ Med 2006; 9: 110-26. 